\title{
Why Is It That the Biggest Resistance With Fighting the Battle Against Bacterial Resistance Seems to Fall on Dermatology Clinicians?
}

\author{
James Q. Del Rosso, DO
}

7 his discussion focuses on antibiotic resistance in acne therapy but also includes general principles related to this subject. "Seeing is believing" is a concept we have all heard many times, and we generally can all agree with and relate to what this is saying to us. However, it is harder to get a consensus of agreement on concepts that are happening beneath the surface but are not visibly apparent. Antibiotic resistance is a concept that falls into this latter category, especially in acne treatment. Many clinicians are not convinced antibiotic resistance is clinically relevant, exclaiming "I just do not see it in my practice." The problem is-especially in the case of acne where oral tetracycline agents commonly are prescribed-how does the clinician "see" antibiotic resistance? Clinicians do not obtain bacterial cultures or perform sensitivity testing as they might do when evaluating a suspected cutaneous infection such as folliculitis, an inflamed postsurgical wound, a purulent leg ulcer, or an abscess. Additionally, if the selected therapy is not as effective as anticipated, it may be attributed to the patient needing another type of treatment or something "stronger," or maybe they are not fully compliant. In fact, a very possible reason for inadequate therapeutic response may be that the predominant Cutibacterium acnes strains in a particular case are proinflammatory, and many of the strains are not highly sensitive to the chosen antibiotic. ${ }^{1}$

In the United States, antibiotic resistance in $C$ acnes is most prevalent with erythromycin, followed by clindamycin, tetracycline, doxycycline, and minocycline, respectively. ${ }^{2}$ The relative patterns of antibiotic resistance in specific geographic regions correlate with the magnitude of specific antibiotic use, and that consistent reduction in use of a given antibiotic in a community can reverse the prevalence of resistance to that antibiotic progressively over time. ${ }^{3}$ Combination therapy approaches to mitigate emergence of resistant bacteria during acne treatment with an exit plan explained up-front with the patient are important to reduce prolonged use or repeated cycles of antibiotic use and in some cases to circumvent antibiotic use and incorporate a different therapeutic approach. ${ }^{1-3}$ Interestingly, in a retrospective chart review of acne patients who were eventually treated with oral isotretinoin at dermatology practices within a major university health system, approximately two-thirds received oral antibiotics for 6 months or longer and one-third for 1 year or longer. ${ }^{4}$ It is easy for all of us to have good intentions; however, in reality it is not always easy, practical, or in the best interest of the patient to stringently enforce recommendations that are determined not to be the best option at that time. Patients get a vote, too, as long as they are fully informed of benefits vs risks.

The concern about emergence of less-sensitive bacteria during acne antibiotic treatment is not limited to discussion of $C$ acnes resistance. Use of both oral and topical antibiotics creates "ecologic mischief," which is the emergence of less-sensitive strains of other bacteria exposed to the antibiotic-both commensal and opportunistic-especially at anatomic sites such as the skin, nasopharyngeal region, and gastrointestinal and genitourinary tracts. ${ }^{5-7}$ Application of topical

From JDR Dermatology Research LLC/Thomas Dermatology, Las Vegas and Henderson, Nevada.

Dr. Del Rosso has served as an advisor, consultant, and speaker for Almirall; Bausch Health (Ortho Dermatologics); EPI Health; Foamix (Menlo Therapeutics); Galderma Laboratories, LP; and Mayne Pharma Group. He also has served as a research investigator for Almirall; Bausch Health (Ortho Dermatologics); Foamix (Menlo Therapeutics); and Galderma Laboratories, LP.

Correspondence: James Q. Del Rosso, DO (jqdelrosso@yahoo.com). 
erythromycin to the face can induce erythromycin-resistant bacteria such as staphylococci on the face as well as at remote sites such as the nares (nasal vestibule) and the back. ${ }^{6}$ Antibiotics used to treat acne, predominantly oral tetracyclines, showed positive oropharyngeal cultures for Streptococcus pyogenes in 33\% (13/39) of treated patients; among these positive cultures, $85 \%$ $(11 / 13)$ were resistant to at least one tetracycline antibiotic. ${ }^{7}$ Importantly, the streptococcal colonization of the oropharynx in individuals taking an oral antibiotic for acne may not induce a clinically apparent pharyngitis in that individual, but that person can carry and spread that streptococcal pathogen to others. In either case, the dermatology clinician, even if he/she suspects the connection related to antibiotic selection pressure and resistance, would not "see" the antibiotic resistance, as the individuals who develop a "sore throat" or strep throat do not seek care for this problem through a dermatology office.

The first formally organized and independent group in dermatology to address antibiotic use and resistance issues was the Scientific Panel on Antibiotic Use in Dermatology, which I put together in 2004 with James J. Leyden, MD (Philadelphia, Pennsylvania) and Guy F. Webster, MD (Hockessin, Delaware), and was comprised mostly of interested dermatologists with contributions from microbiologists and infectious disease specialists. A series of meetings, publications, and presentations have emerged from this group, which now falls under the auspices of the American Acne \& Rosacea Society. Through the efforts of these organizations and other groups and companies with a strong interest in combating antibiotic resistance, we continue to see slow but steady progress in enlightening dermatology clinicians to think about if and when antibiotic therapy is needed and for how long. The subject of when antibiotics are not necessary also has been addressed, including both oral and topical antibiotics in many common scenarios encountered in dermatology practice. ${ }^{8}$ Examples include incision and drainage of an inflamed epidermal cyst without antibiotic therapy and use of white petrolatum instead of a topical antibiotic after most dermatologic procedures such as biopsies, tangential procedures, and closures after excisional procedures. Overall, the potential for topical antibiotics containing bacitracin and/or neomycin to induce allergic contact dermatitis is higher than the risk for postoperative wound infection. A major reason to avoid facilitating the emergence of antibioticresistant bacteria is that these organisms are efficient in packaging their resistance genes along with those from other bacteria, thus creating multidrug-resistant bacterial strains. This situation creates bigger challenges with trying to select effective therapies.

A cross-sectional analysis of antibiotics prescribed by dermatologists from January 1, 2008, to December 31, 2016, performed via a large commercial prescription claims database showed that among almost 1 million courses of oral antibiotics prescribed by approximately 12,000 unique dermatology prescribers, overall antibiotic prescribing decreased $36.6 \%$, reflecting a drop of 1.23 courses per 100 visits, with much of the reduction occurring among extended antibiotic courses for acne and rosacea. ${ }^{9}$ Dermatology clinicians appear to be increasing their consideration of treatment alternatives such as oral spironolactone in adult female patients or earlier transition to oral isotretinoin therapy before starting another cycle with the same or a different oral antibiotic. Some have increased the use of physical device therapies. Importantly, we do not want to throw out the baby with the bathwater. Oral antibiotics remain important agents for treatment of moderate to severe inflammatory acne and in rosacea when subantibiotic-dose doxycycline is not accessible or is not effective after an adequate trial of therapy. Last but not least, a full-court press with an optimal topical regimen is the foundation of acne therapy, as monotherapy with an oral antibiotic for acne is considered dermatologic heresy and for good reason.

\section{REFERENCES}

1. Del Rosso JQ, Leyden JJ. Status report on antibiotic resistance: implications for the dermatologist. Dermatol Clin. 2007;25:127-132.

2. Leyden JJ, Del Rosso JQ, Webster GF. Clinical considerations in the treatment of acne vulgaris and other inflammatory skin disorders: a status report. Dermatol Clin. 2009;27:1-15.

3. Del Rosso JQ, Zeichner JA. The clinical relevance of antibiotic resistance: thirteen principles that every dermatologist needs to consider when prescribing antibiotic therapy. Dermatol Clin. 2016;34:167-173.

4. Nagler AR, Milam EC, Orlow SJ. The use of oral antibiotics before isotretinoin therapy in patients with acne. J Am Acad Dermatol. 2016;74:273-279.

5. Del Rosso JQ, Kim GK. Topical antibiotics: therapeutic value or ecologic mischief? Dermatol Ther. 2009;22:398-406.

6. Bowe WP, Leyden JJ. Clinical implications of antibiotic resistance: risk of systemic infection from Staphylococcus and Streptococcus. In: Shalita AR, Del Rosso JQ, Webster GF, eds. Acne Vulgaris. London, England: Informa Healthcare; 2011:125-133.

7. Levy RM, Huang EY, Roling D, et al. Effect of antibiotics on the oropharyngeal flora in patients with acne. Arch Dermatol. 2003;139:467-471.

8. Hirschmann JV. When antibiotics are unnecessary. Dermatol Clin. 2009;27:75-83.

9. Barbieri JS, Bhate $\mathrm{K}$, Hartnett KP, et al. Trends in oral antibiotic prescription in dermatology, 2008 to 2016. JAMA Dermatol. 2019;155:290-297. 